\title{
The Path Planning of Agricultural AGV in Potato Ridge Cultivation
}

\author{
Tongning Mai, Shiquan Shao*, Zehui Yun \\ Southwest Minzu University, Chengdu Sichuan, 610041 China \\ Email: mai_8717@163.com
}

\begin{abstract}
In order to ensure that the agricultural AGV spend least time in the multi-point monitoring process, this paper analyzes the characteristics of the ridge farmland and proposes a path planning algorithm in the ridge farmland based on ant colony algorithm. The actual distance between each monitoring point is discussed in detail. The problem of path planning of multi-point measurement in ridge farmland is transformed into TSP problem. By establishing a suitable model and using Matlab to simulate the path planning algorithm, the results show that the algorithm is feasible.
\end{abstract}

Keywords: Path planning, ant colony algorithm, agricultural AGV, precision agriculture

\section{Introduction}

As one of the world's four major food crops, potato has high nutritional value, strong adaptability and large yield, which has been widely planted in China. In potato cultivation, the moisture content in soil has a great influence on each period of the potato. Therefore, improving the utilization of water resources during the potato planting is one of the main means to increase the yield and quality of the potato[1]. Precision irrigation is an important part of precision agriculture. Maximizing the ratio of benefit-cost in large-scale farmland is one of the main objectives of precision irrigation. Precise monitoring of soil moisture is a prerequisite to achieve precision irrigation. Due to the spatial variation of crop growth and yield caused by changes in topography, slope, and soil physical properties [2], it is extremely important to monitor soil moisture reasonably. Studies have shown that available water holding capacity (AWC) reflects soil water carrying capacity[3], and apparent electrical conductivity (ECa) is the difference of soil structure and water content [4].Therefore, according to the AWC and ECa of the soil, the prescription map of the water holding capacity in farmland can be drawn. The whole farmland can be statically partitioned by the prescription map to realize the variable irrigation[5]. A monitoring point is set in a narrow region where the water holding capacity is similar according to the prescription map. The farmland in different regions will be precisely irrigated according to the information of soil moisture of the monitoring point. Using agricultural AGV to collect the information of soil moisture from different monitoring points can realize variable irrigation of whole farmland and greatly improve work efficiency [6].

Due to the large number of monitoring points in large-scale farmland, in order to reduce working time and driving distance of agricultural AGV , it is necessary to carry out reasonable path planning. Timo Oksanen discusses a coverage path planning problem in the case of agricultural fields and agricultural machines. Methods and algorithms to solve this problem are developed. The necessary condition is to cover the whole field, and the goal is to find as efficient a route as possible[7]. R. González et al. studied navigation methods for mobile agricultural robots in greenhouses and have been implemented and tested in a real environment, with promising results [8]. Li Qiaojun et al. realized a set of path planning and real-time positioning system for agricultural robots based on data mining by using particle filter algorithm[9]. Stoll proposed to optimize the path of farmland by dividing it into several plots according to the longest edge of farmland[10]. Pratap introduce a new path planning problem and present constant-factor approximation algorithms, which can find a path minimizing both travel and measurement time by using the UAV+UGV system[11]. All path planning algorithms above were established in the case that agricultural AGV can walk freely in the farmland. But because of the ridge, agricultural AGV cannot travel across the ridge and only travel in the furrow along the direction of the ridge. So the algorithm above is not applicable to the path planning problem of ridge farmland. Moreover, most of the algorithms above are about the coverage path planning problem of farmland. It is not necessary to traverse the entire farmland but only travel 
several monitoring point in ridge farmland. Traditionally, the problem of Multi-point travelling can be abstracted into the Traveling Salesman Problem (TSP). However, in the ridge farmland, the agricultural AGV only can drive through the narrow path between the ridges. Therefore, the path planning for ridge cropland is a variant of TSP problem. There is no reasonable path planning method for agricultural AGV in ridge[12]. According to the characteristics of ridge farmland, this paper analyzes the TSP problem in ridge farmland, uses the positive feedback and global optimization of ant colony algorithm to realize the path planning of Multi-point travelling in ridge. it can reduce total measurement times improve work efficiency and save energy.

\section{$2 \quad$ Model of Potato Farmland}

Potato cultivation requires lots of water and high soil moisture. If the seed of potato is dry, the potato production will decrease; if the rain increases, the soil moisture will increase, which will cause the production to decrease and result in the rotten seed during storage. Potatoes are usually grown on ridges for raising the ground temperature and preventing drought as the figure 1 shows. In general, the path planning of multi-point travelling can be regarded as a classic TSP problem. The reasonable path planning can be performed by using simulated annealing algorithm, genetic algorithm, and ant colony algorithm. Since the agricultural AGV travels between the ridges for multi-point travelling, in this case, the AGV cannot travel across the ridges. So the ordinary TSP problem solution is not applicable. Therefore, this paper improves the ant colony algorithm according to the characteristics of ridge farmland. The agricultural AGV can measure soil moisture of multiple points in the shortest path in the ridge farmland, which saves the measurement time and electricity.

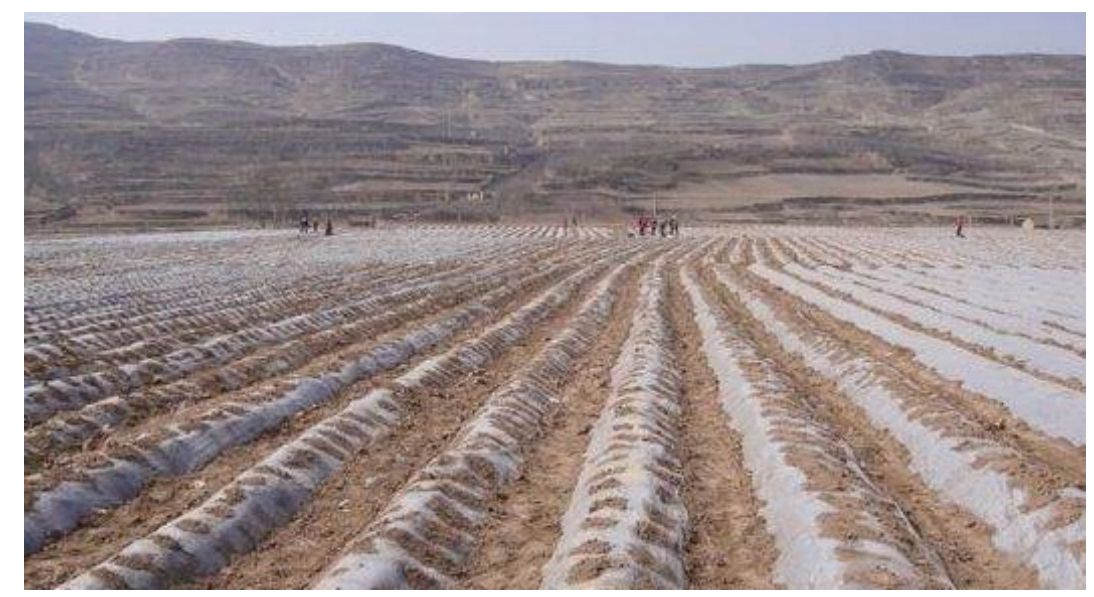

Figure 1. Ridge planting of potatoes

Figure 2 shows the ridge planting model of potato. The shaded area is the ridge. The potatoes are planted on each ridge. Between the two ridges is furrow. No crops are planted. The agricultural AGV travels in each furrow. The monitoring points are distributed on each ridge. Since the agricultural AGV only can travel in the furrow, the position parallel to the monitoring point in the right furrow is selected as the parking point. The path planning problem of the monitoring point is transformed into the path planning of the parking spot. 


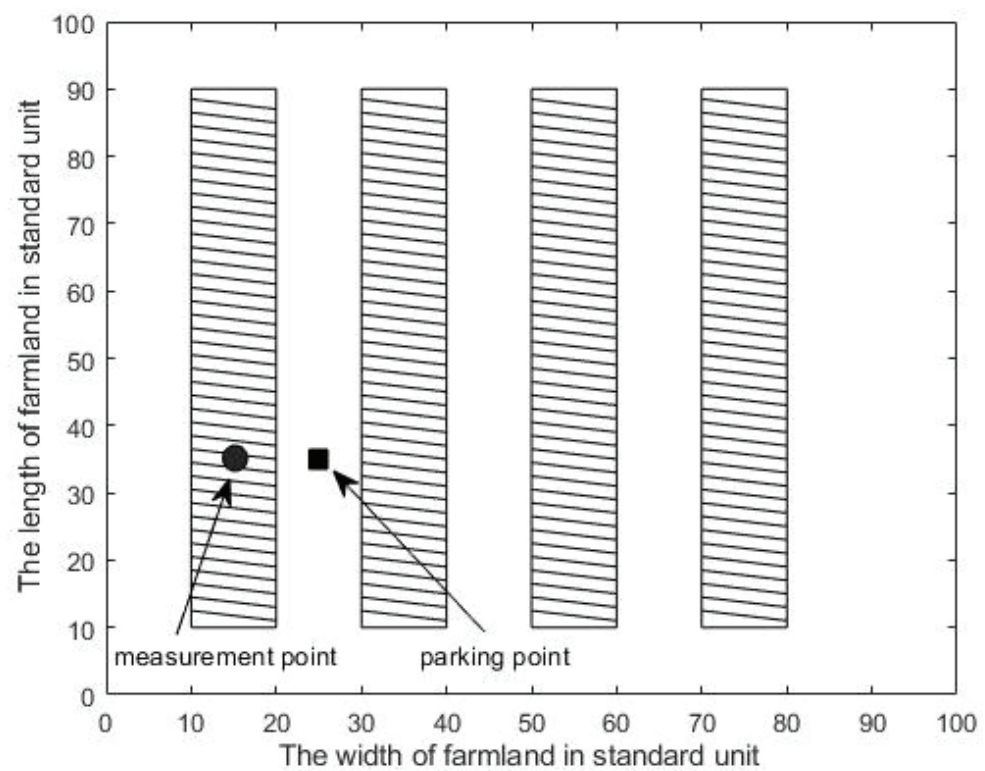

Figure 2. Ridge planting model of potato

In the ridge cultivation, the whole farmland can be divided into several plots to facilitate the field management. No crops are planted between each plot, and agricultural AGV can pass through the free area between plots. Therefore, the agricultural AGV can travel in furrows or in the free area between plots. As shown in Fig. 3, the path planning of multi-point traveling in the ridge farmland can be divided into two cases.

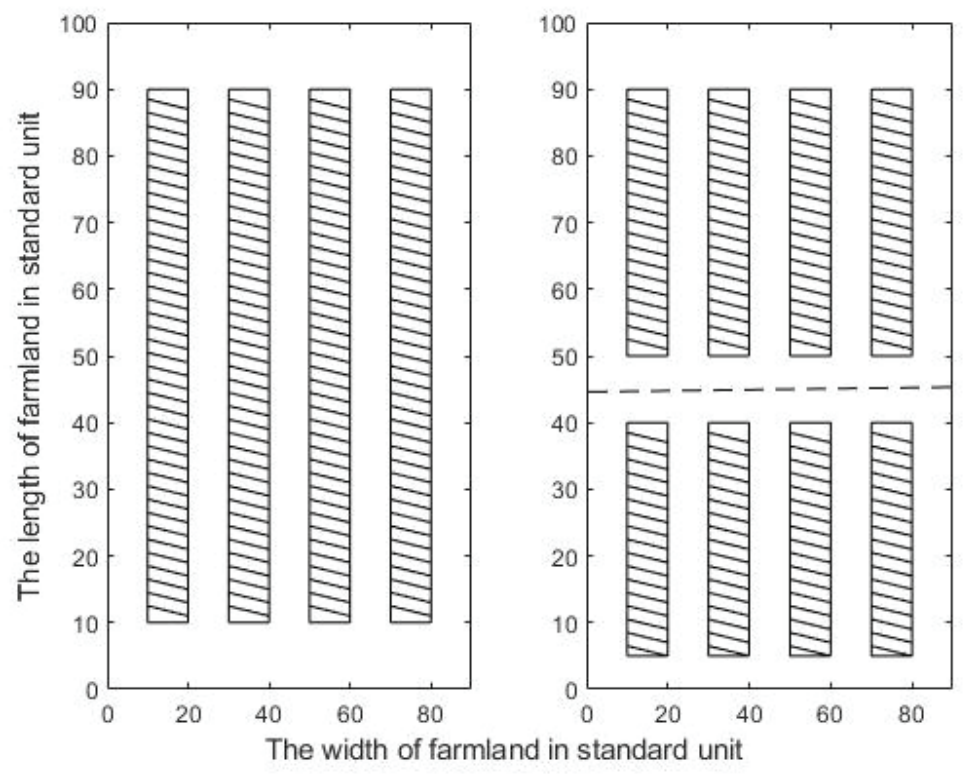

Figure 3. Two types of ridge farmland

a) There is only one plot of the whole farmland, and the agricultural AGV only can travel in furrows or in the free area at the top and bottom of the farmland.

b) The whole farmland is divided into multiple plots. There is a free zone between each plot. As shown in Figure 3, the farmland is divided into two plots by the dotted line. In addition to driving in furrow or on the top and bottom of the farmland, the AGV also can travel along the dotted line between two plots. 


\section{Ant Colony Algorithm}

The ant colony algorithm was proposed by the Italian scholar Dorigo et al. There are $\mathrm{n}$ parking points. According to the ant colony algorithm, $\mathrm{P}_{\mathrm{ij}}^{\mathrm{k}}(\mathrm{t})$ represents the probability that the ant $k$ moves from the parking point $i$ to the point $j$, then

$$
P_{i j}^{k}(t)=\left\{\begin{array}{c}
\frac{\left[\tau_{i j}(t)\right]^{\alpha}\left[\eta_{i j}\right]^{\beta}}{\sum_{s \in \text { allowed }_{k}\left[\tau_{i s}(t)\right]^{\alpha}\left[\eta_{i s}\right]^{\beta}}, j \in \text { allowed }_{k}} \\
0, \text { otherwise }
\end{array}\right.
$$

$\tau_{\mathrm{ij}}(\mathrm{t})$ is the pheromone concentration from point $i$ to point $j$ at time $t$. $\eta_{\mathrm{ij}}$ is the heuristic function, indicating the expected degree of ant from point $i$ to point $j$, and allowed $_{\mathrm{k}}$ is the parking point the ant $k$ next choose. $\alpha$ and $\beta$ are pheromone heuristic factors and expected heuristic factors, respectively.

$$
\eta_{i j}=\frac{1}{d_{i j}}
$$

For ridge planting, agricultural AGV only travels in free areas and cannot pass directly between ridges. In ridge farmland, the distance between each parking spot cannot be expressed by Euclidean distance. The distance between the two points should be the actual driving distance of the agricultural AGV.

As shown in Figure 4, the two parking spots are located on the same plot in farmland. There are two paths from the first to the next.

Path 1: The AGV arrives at the top of the plot along the positive direction of the Y-axis from the first parking spot, then passes from the top free zone to the next furrow. Finally, the AGV arrive at the second parking spot.

Path 2: The AGV arrives at the bottom of the plot along the negative direction of the Y-axis from the first stop point, and passes from the bottom free zone to the furrow. Finally, the AGV arrive at the second parking point.

If the center line of the free area at the bottom and top of the plot is $y_{k}, y_{k+1}$ and the coordinates of the two parking points are $\left(\mathrm{x}_{\mathrm{i}}, \mathrm{y}_{\mathrm{i}}\right)$ and $\left(\mathrm{x}_{\mathrm{j}}, \mathrm{y}_{\mathrm{j}}\right)$, the actual distance between the two points is:

$$
\begin{gathered}
d_{i j} 1=\left|y_{k}-y_{i}\right|+\left|y_{k}-y_{j}\right|+\left|x_{i}-x_{j}\right| \\
d_{i j} 2=\left|y_{k+1}-y_{i}\right|+\left|y_{k+1}-y_{j}\right|+\left|x_{i}-x_{j}\right| \\
d_{i j}=\min \left(d_{i j} 1, d_{i j} 2\right)
\end{gathered}
$$

The shortest path of two paths above is the actual distance the AGV travels at two points if the two parking points are located on the same plot in farmland.

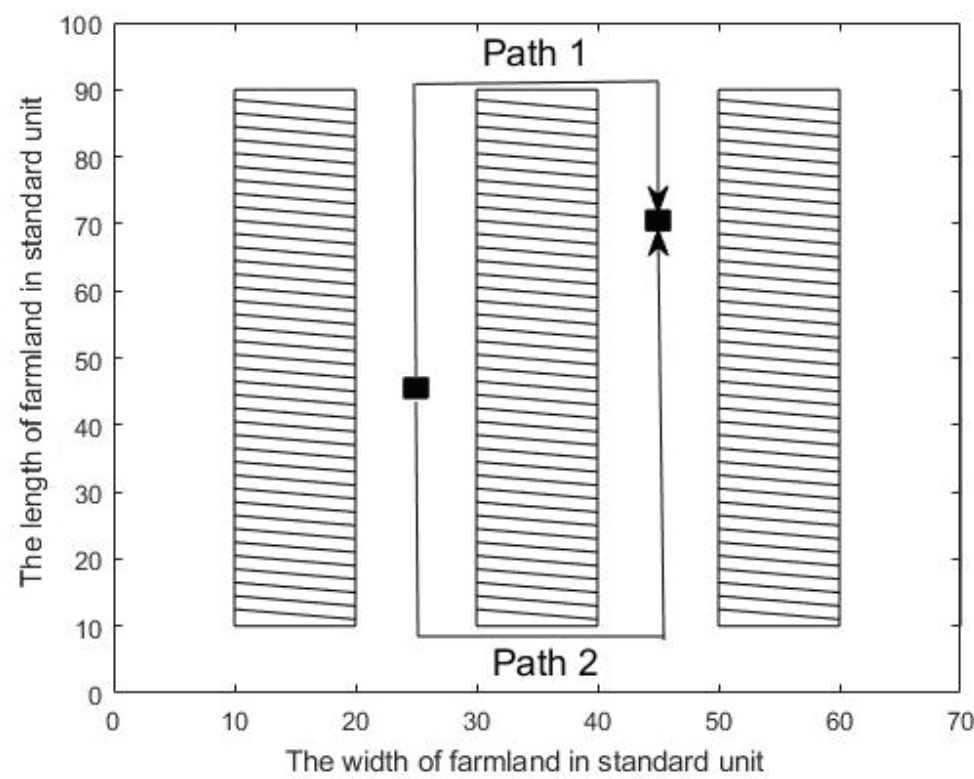

Figure 4. Path in single plot of ridge farmland 
As shown in Fig. 5, the two points are located on different plots in farmland. the AGV can travel along the free area between the two plots. The shortest path from one point to the next is:

$$
\mathrm{d}_{\mathrm{ij}}=\left|\mathrm{y}_{\mathrm{i}}-\mathrm{y}_{\mathrm{j}}\right|+\left|\mathrm{x}_{\mathrm{i}}-\mathrm{x}_{\mathrm{j}}\right|
$$

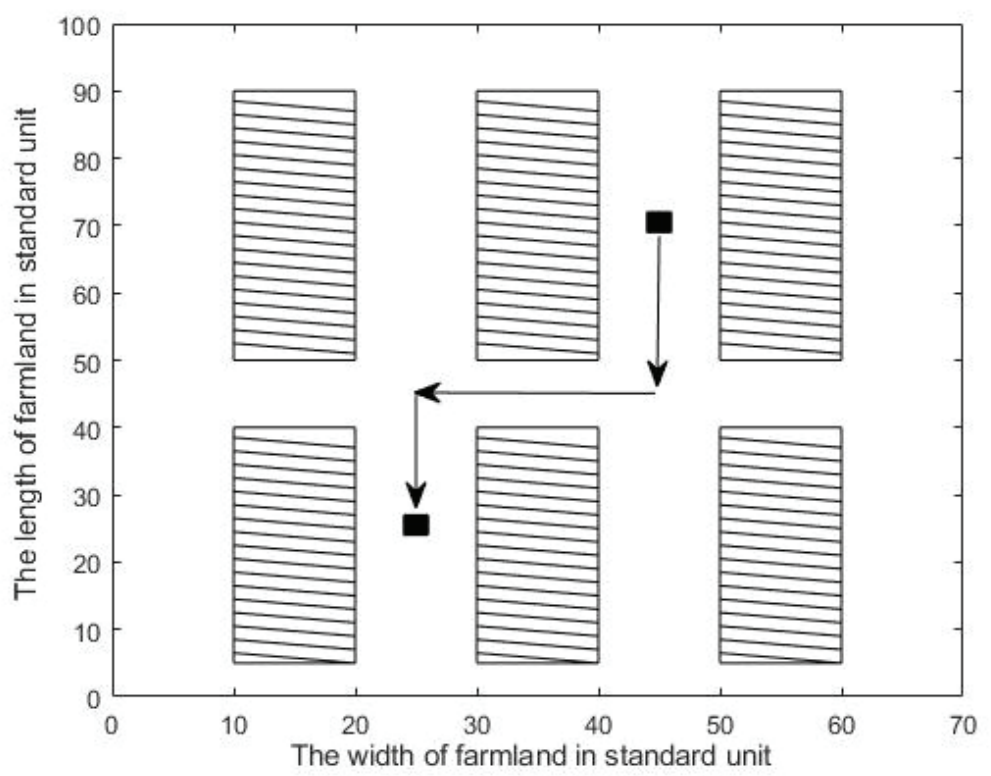

Figure 5. Path in different plot of ridge farmland

Therefore, $\mathrm{d}_{\mathrm{ij}}$ should be the real distance traveled by the AGV. For the ridge farmland, if the whole farmland is divided into $\mathrm{m}$ plots, the dividing line is $\mathrm{y}=\mathrm{y}_{\mathrm{k}}, k \in[1, m-1]$. If the coordinates of point $i$ and point $j$ are $\left(\mathrm{x}_{\mathrm{i}}, \mathrm{y}_{\mathrm{i}}\right)$ and $\left(\mathrm{x}_{\mathrm{j}}, \mathrm{y}_{\mathrm{j}}\right)$, then:

1) if $\mathbf{x}_{\mathbf{i}}=\mathbf{x}_{\mathbf{j}}$, that means point $i$ and point $j$ are in the same ridge, then:

$$
\mathrm{d}_{\mathrm{ij}}=\sqrt{\left(\mathrm{y}_{\mathrm{i}}-\mathrm{y}_{\mathrm{j}}\right) 2}
$$

2) If $\mathrm{x}_{\mathrm{i}} \neq \mathrm{x}_{\mathrm{j}}$, and $\mathrm{y}_{\mathrm{k}}<\mathrm{y}_{\mathrm{j}}, \mathrm{y}<\mathrm{y}_{\mathrm{k}+1}$, then point $i$ and point $j$ are in the same plot in farmland but not in the same ridge. The real distance from the point $i$ and point $j$ is:

$$
\left\{\begin{array}{c}
d_{i j} 1=\sqrt{\left(y_{k}-y_{i}\right)^{2}+\left(y_{k}-y_{j}\right)^{2}+\left(x_{i}-x_{j}\right)^{2}} \\
d_{i j} 2=\sqrt{\left(y_{k+1}-y_{i}\right)^{2}+\left(y_{k+1}-y_{j}\right)^{2}+\left(x_{i}-x_{j}\right)^{2}} \\
d_{i j}=\min \left(d_{i j} 1, d_{i j} 2\right)
\end{array}\right.
$$

3) If none of the conditions above are met, then point $i$ and point $j$ are on different plots. The real distance from the point $i$ and point $j$ is:

$$
d_{i j}=\sqrt{\left(x_{i}-x_{j}\right)^{2}+\left(y_{i}-y_{j}\right)^{2}}
$$

When the ant completes a loop, the update rule of pheromone on each path is calculated according to formula (10).

$$
\begin{gathered}
\tau_{\mathrm{ij}}(t+1)=(1-\rho) \tau_{\mathrm{ij}}(t)+\Delta \tau_{\mathrm{ij}}(\mathrm{t}) \\
\Delta \tau_{\mathrm{ij}}^{\mathrm{k}}(\mathrm{t})=\left\{\begin{array}{c}
\frac{\mathrm{Q}}{\mathrm{L}_{\mathrm{K}}}, \quad(\mathrm{t})=\sum_{\mathrm{k}=1}^{\mathrm{m}} \Delta \tau_{\mathrm{ij}}^{\mathrm{k}}(\mathrm{t}) \quad \in \mathrm{p}_{\mathrm{k}}(\text { begin, end }) \\
0, \text { otherwise }
\end{array}\right.
\end{gathered}
$$

where $\rho$ is the degree of pheromone volatilization. $\Delta \tau_{\mathrm{ij}}^{\mathrm{k}}(\mathrm{t})$ represents the pheromone left by the $k$ th ant between point $i$ and point $j . \Delta \tau_{\mathrm{ij}}(\mathrm{t})$ is the increment of pheromone from point $i$ to point $j$ in this loop. 
$\Delta \tau_{\mathrm{ij}}^{\mathrm{k}}(\mathrm{t})$ is calculated by the formula (12), where $Q$ is a constant, which is the total amount of pheromone released by the ant in one loop, and $\mathrm{L}_{\mathrm{K}}$ is the total length of the ant $k$ walking.

\section{The References Section Simulation Results and Analysis}

In order to facilitate the simulation analysis of the path planning algorithm in ridge farmland, according to the characteristics of ridge farmland, the whole farmland is mapped to the $1000 * 200$ coordinate system for modeling analysis. Because the ridge farmland also can be managed by in plots, the experiment is divided into three groups:

1) There is only one plot of the whole farmland. The agricultural AGV only can pass through on the top or bottom of the entire farmland except for travelling along the furrow.

2) The entire farmland is divided into 2 plots. The AGV also can pass through the free area between the two plots, increasing the lateral freedom of the AGV in the ridge field.

3) The farmland is divided into three plots. There are two horizontal free areas in the farmland, which further increase the lateral freedom of the AGV.

When there is only one plot in the whole farmland, Figure 6 shows the shortest path of the AGV that travelled all points in turn according to the improved ant colony algorithm. The order of travelling all point is identified in the figure. The connection between each point in the figure is the driving path of the AGV. Figure 7 shows the average distance and the shortest distance that ants travelled all points in the iteration. The experimental result shows that with the increase of iterations, the average distance and the shortest distance of the AGV continuously decrease until it is stable. In the single plot, the shortest driving distance of the agricultural AGV travelling through all points is 6260 unit length. If the AGV travels through all the points by traversing through all furrows, it needs to walk 7600 unit length. Using this algorithm, the walking distance of the AGV is $82.4 \%$ of the distance by traversing through all furrows. Therefore, the algorithm can reduce the driving distance of the AGV in a single plot, achieve the purpose of improving efficiency and saving power. But the effect is not particularly remarkable.

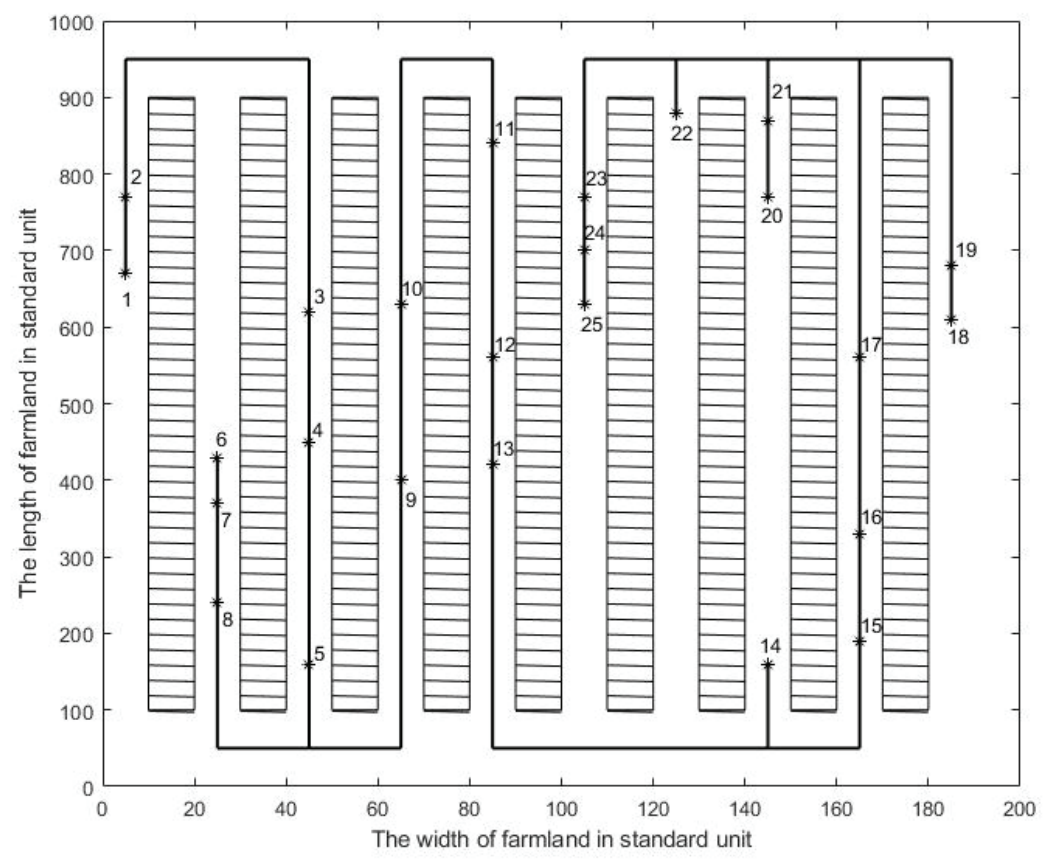

Figure 6. Shortest path planning for single plot in ridge farmland 


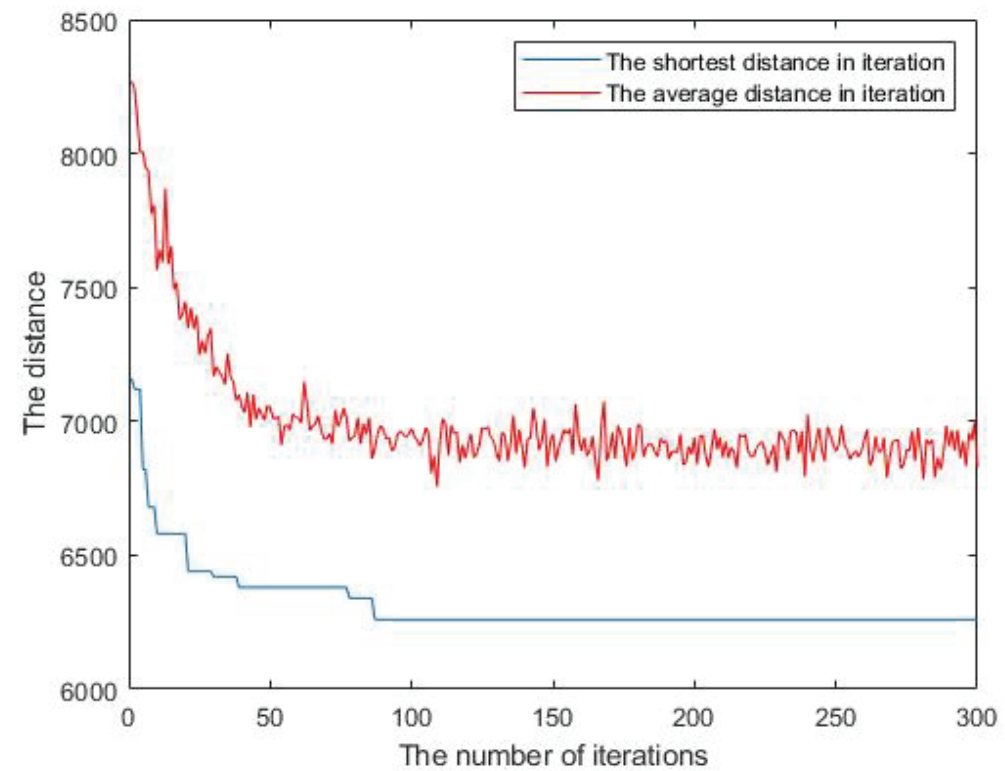

Figure 7. Average distance and shortest distance in iteration in single plot

Figure 8 shows the shortest path when the farmland is divided into 2 plots. In the standardized coordinate system, the farmland is divided into two parts by $\mathrm{y}=500$. The area between $\mathrm{y}=450$ and $\mathrm{y}=550$ belongs to the free area. The AGV also can travel from $\mathrm{y}=500$. As the whole farmland is divided into two plots, the freedom of the AGV to travel in the lateral direction has been improved to a certain extent. Therefore, the shortest distance actually travelled by the AGV is further reduced as shown in Figure 9. In this experiment, the driving distance of the AGV dropped to 4380 unit length. The driving distance is $70 \%$ of the driving distance in single plot, $57.6 \%$ of the distance by traversing through all furrows. The overall efficiency of the AGV has been further improved.

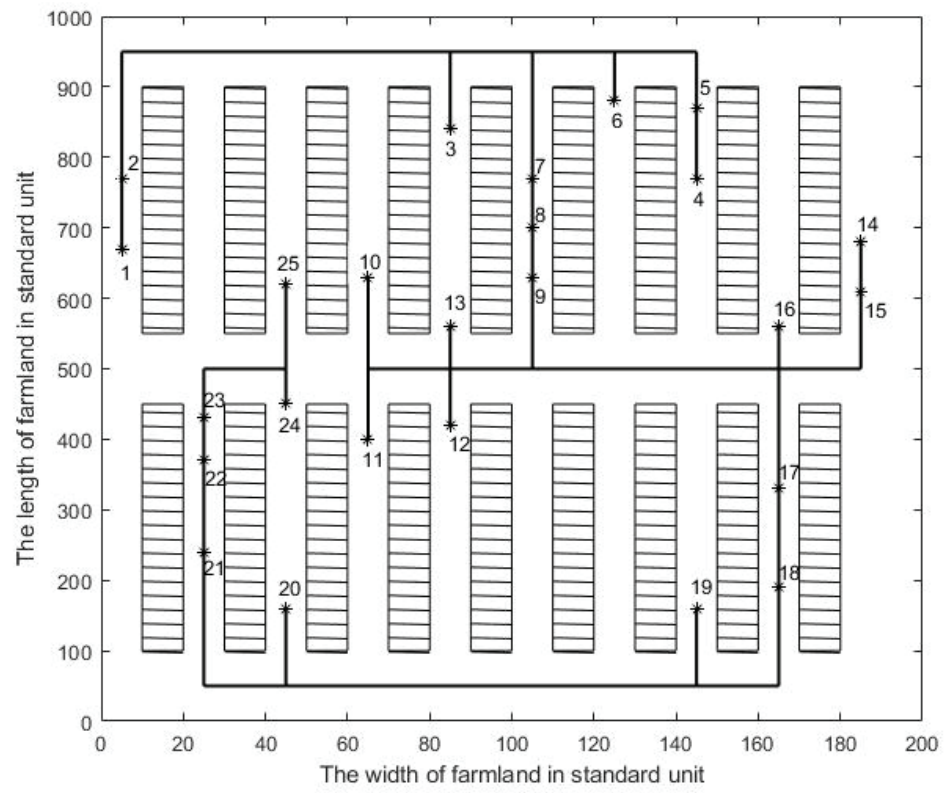

Figure 8. Shortest path planning for two plots in ridge farmland 


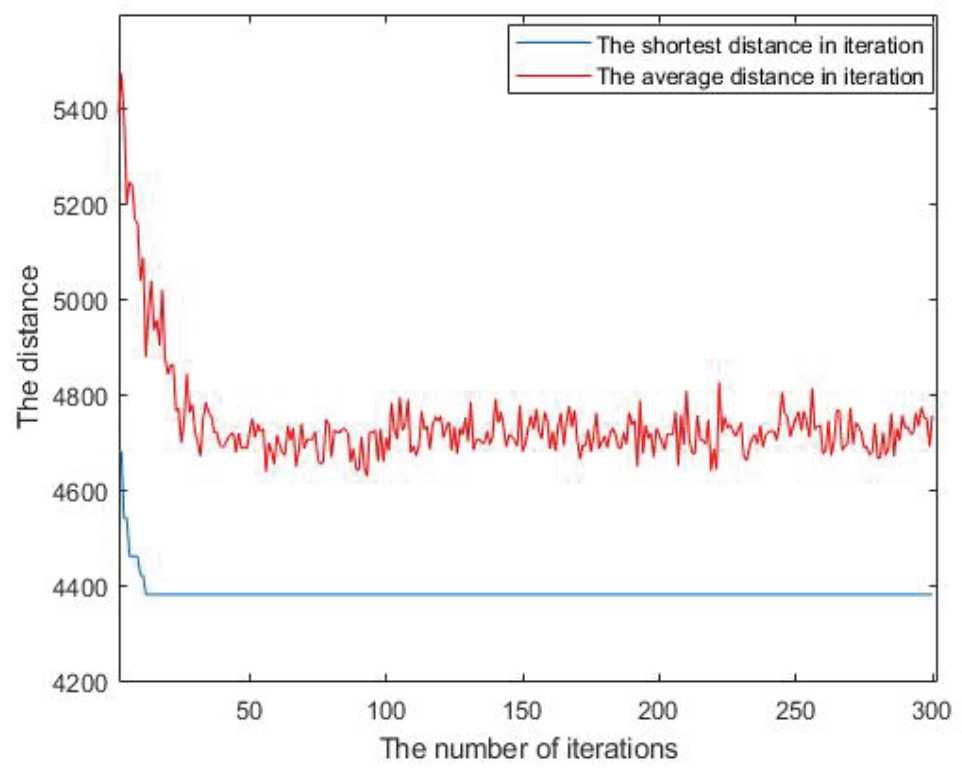

Figure 9. Average distance and shortest distance in iteration in two plots

As shown in Figure 10, the whole farmland is divided into three plots. In the standardized coordinate system, the farmland is divided into three parts by $\mathrm{y}=350$ and $\mathrm{y}=650$, where $\mathrm{y}=340$ to $\mathrm{y}=360$ and $\mathrm{y}=640$ to $\mathrm{y}=660$ are free areas, the AGV also can pass in the direction of $\mathrm{y}=350$ and $\mathrm{y}=650$. In this experiment, the farmland is divided into three parts. The lateral driving freedom of the agricultural AGV has been improved again. From figure 11, the shortest driving distance of the AGV is further reduced, from 4440 unit length in two plots to 3500 unit length. The driving distance is $55.9 \%$ of one plot, $46.1 \%$ of the distance by traversing through all furrows. Therefore, the algorithm can minimize the driving distance of the agricultural AGV and improve the travelling efficiency.

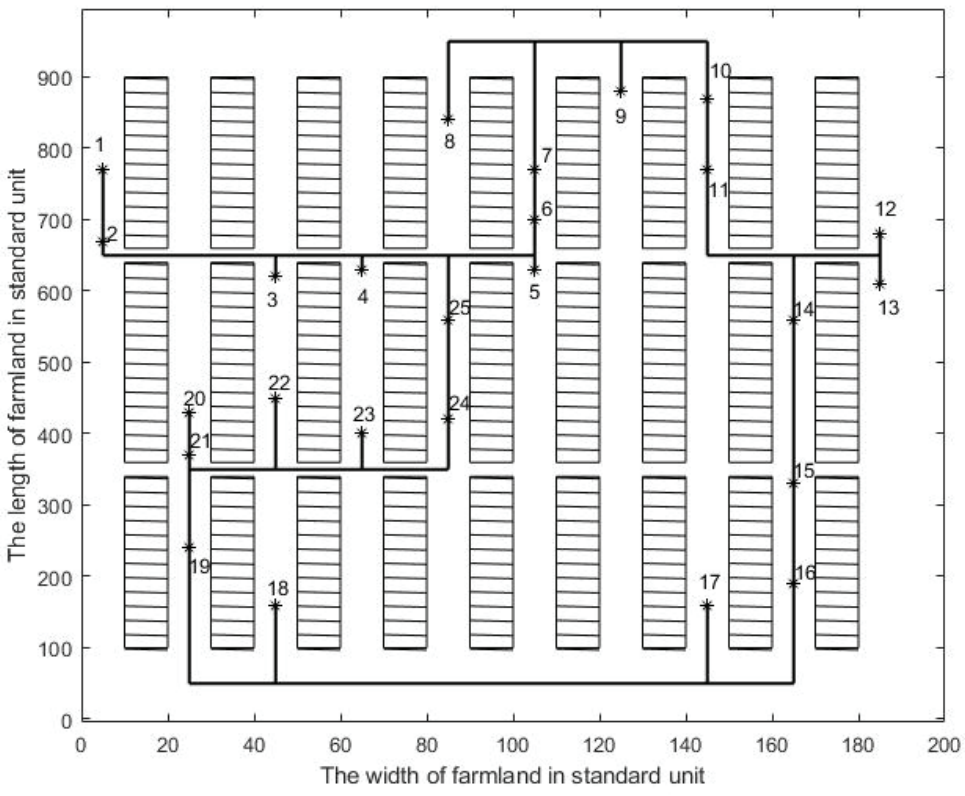

Figure 10. Shortest path planning for three plots in ridge farmland 


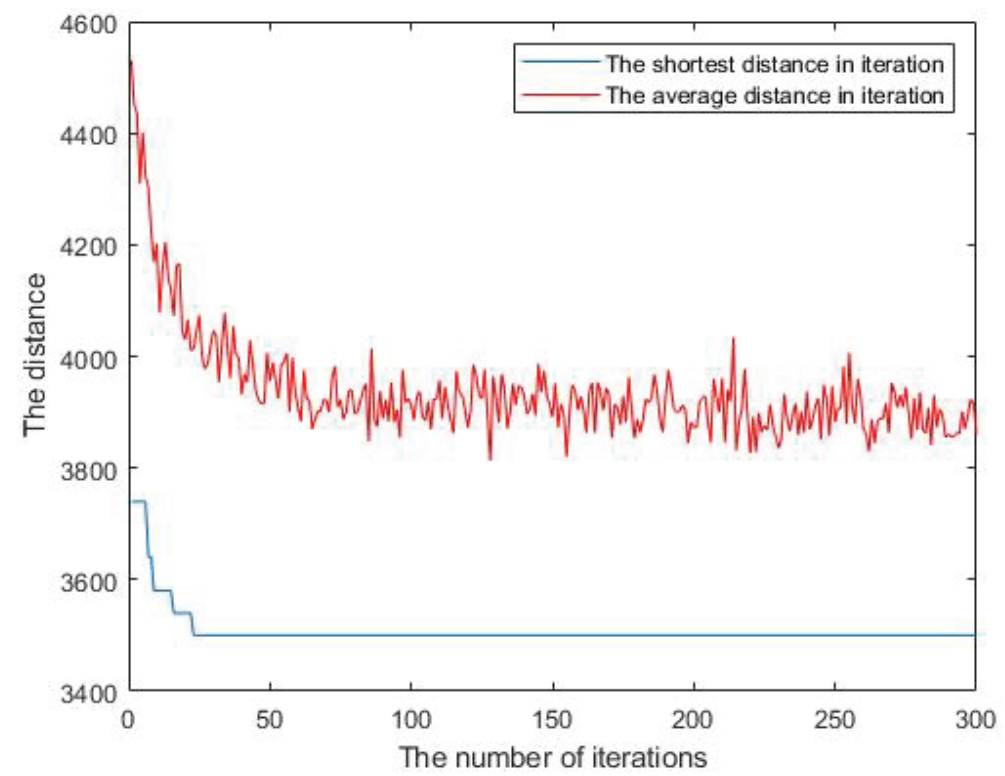

Figure 11. Average distance and shortest distance in iteration in three plots

Table1. Analysis of three simulation experiments

\begin{tabular}{ccc}
\hline Farmland type & Actual travel distance & \begin{tabular}{c} 
Actual travelling distance \\
\cline { 3 - 3 }
\end{tabular} \\
\hline Single plot in farmland & 6260 & $82.4 \%$ \\
Two plots in farmland & 4380 & $57.6 \%$ \\
Three plots in farmland & 3500 & $46.1 \%$ \\
\hline
\end{tabular}

Table 1 is the analysis table of three simulation experiments. It can be seen from the table that in the path planning problem of ridge farmland, using path planning algorithm based on ant colony algorithm can greatly reduce the distance of agricultural AGV travelled in the farmland. it can improve the working efficiency of agricultural AGV, and effectively reduce the energy consumption.

In addition, it is found that in the path planning of the ridge farmland, the number of plots in farmland has a huge impact on the path planning through the comparison of three simulation experiments. The more plots there is in farmland, the higher degree of lateral freedom of the AGV, the shorter travelling distance, and the higher efficiency of the algorithm. But the specific impact of the number of plots on the algorithm needs further study.

\section{Conclusion}

For potato planting, it is necessary to accurately monitor the soil moisture in the farmland. The monitoring point is located in different locations of the farmland, and agricultural AGV are needed to measure the soil moisture in turn. Because agricultural AGV only can travel between ridges but not cross ridges, this paper proposes a path planning algorithm for ridge farmland based on ant colony algorithm according to the characteristics of ridge farmland. The ridge farmland was divided into single plot farmland and multi-plot farmland. The actual driving distance of agricultural AGV in ridge farmland was studied in detail. The path planning algorithm of agricultural AGV in ridge farmland is simulated by the software of Matlab. The results of three groups of experiment show that the algorithm is feasible for both models. It enables agricultural AGV to travel all monitoring points in farmland with the shortest driving distance. In addition, the comparison of the three sets of experimental data shows that as the number of farmland plots increased, the lateral freedom of the trolley also increased, the efficiency of the algorithm is higher, and the distance traveled by the AGV is further reduced. 
Acknowledgements. The work of this paper is supported by the basic research business fee of the Central University of the Southwest Minzu University (Project number: 2018YXXS26). A special acknowledgement should give to Southwest Minzu University for its experimental conditions and technical support.

\section{References}

1. Wang Ya-hong,et al. "Effects of different planting patterns on soil temperature and w ater use efficiency of potato in arid regions of Gansu Province." Journal of Gansu Agricultural University,2009,44(06):19-23+43.

2. Zhao Weixia, Li Jiusheng, Li Yanfeng. "Review on variable rate irrigation with continuously moving sprinkler machines [J]." Transactions of the Chinese Society of Agricultural Engineering. 2016, 32(13): 1-7.

3. King, Bradley A, J. C. Stark, and Richard W. Wall. "Comparison of site-specific and conventional uniform irrigation management for potatoes." Applied Engineering in Agriculture22.5 (2006): 677-688.

4. Hedley, C. B., et al. "Rapid identification of soil textural and management zones using electromagnetic induction sensing of soils." Soil Research 42.4 (2004): 389-400.

5. Zhao Weixia, Li Jiusheng, Yang Rumiao. "Yields and water-saving effects of crops as affected by variable rate irrigation management based on soil water spatial variation." Transactions of the Chinese Society of Agricultural Engineering,2017,33(02):1-7.

6. Gao Guoqin, Li Ming."Navigating path recognition for greenhouse mobile robot based on K-means algorithm." Transactions of the Chinese Society of Agricultural Engineering. 2014, 30(7): 25-33.

7. Oksanen, T, and A. Visala. "Path Planning Algorithms for Agricultural Machines." Journal of Field Robotics 26.8(2010):651-668

8. R. González, et al. "Navigation Techniques for Mobile Robots in Greenhouses." Applied Engineering in Agriculture 25.2(2009):153-165

9. Li Qiaojun, Liang Junjuan. "Path Planning and Real-time Positioning System for Agricultural Robot-Based on Data Mining Technology." Applied Engineering in Agriculture. 2019,41(06):214-218.

10. Stoll, A., J. Stafford, and A. Werner. "Automatic operation planning for GPS-guided machinery." Proceedings of 4th European Conference on Precision Agriculture. 2003.

11. Tokekar, Pratap, et al. "Sensor planning for a symbiotic UAV and UGV system for precision agriculture." IEEE Transactions on Robotics 32.6 (2016): 1498-1511.

12. Wang Hongjun,Xu Jun,Zhao Hui. " Path Planning of Greenhouse Robot Based on Potential Field- Ant Colony Algorithm." Jiangsu Agricultural Sciences. 2017,45(18):222-225. 